\title{
Development and Assessment of a Data Set Containing Frame Images and Dense Airborne Laser Scanning Point Clouds
}

\author{
Antonio Maria Garcia Tommaselli ${ }^{\circledR}$, Member, IEEE, Maurício Galo ${ }^{(}$, Member, IEEE, Thiago Tiedtke dos Reis, \\ Roberto da Silva Ruy, Marcus Vinicius Antunes de Moraes, and Wander Vieira Matricardi
}

\begin{abstract}
This letter describes the main features of a data set that contains aerial images acquired with a medium format digital camera and point clouds collected using an airborne laser scanning unit, as well as ground control points and direct georeferencing data. The flights were performed in 2014 over an urban area in Presidente Prudente, São Paulo, Brazil, using different flight heights. These flights covered several features of interest for research, including buildings of different sizes and roof materials, roads, and vegetation. Three point clouds with different densities, a block of digital aerial images, and auxiliary data are available. A geometric assessment was conducted to ensure the accuracy and consistency of the data, and an RMSE of $7 \mathrm{~cm}$ was achieved using bundle block adjustment. The data set is freely available for download, and it will be expanded with data collected over time.
\end{abstract}

Index Terms-Airborne laser scanner, data set, digital aerial images, point clouds.

\section{INTRODUCTION}

D ATA sets are a valuable tool for research in many scientific and technological fields. In photogrammetry and remote sensing, most available data sets cover locations in the Northern Hemisphere. Detailed and updated data sets from different sensors are lacking, especially in tropical areas. This situation can be explained by the high cost and complexity of creating, testing, and documenting such data sets. Some scientific societies such as the Geosciences and Remote Sensing Society (GRSS-IEEE) and the International Society for Photogrammetry and Remote Sensing (ISPRS) encourage research groups to publish data sets as a means of supporting research and to enable comparison of the results obtained by applying different methods to standardized data sets. On the other hand, close-range data sets for use in computer vision research are much more common, probably because of the

Manuscript received June 13, 2017; revised August 15, 2017 and November 25, 2017; accepted November 25, 2017. Date of publication December 29, 2017; date of current version January 23, 2018. This work was supported in part by the Foundation for the Development of Unesp (Fundunesp), in part by São Paulo Research Foundation (FAPESP), in part by National Council for Scientific and Technological Development (CNPq), in part by Engemap, and in part by SensorMap. (Corresponding author: Antonio Maria Garcia Tommaselli.)

A. M. G. Tommaselli, M. Galo, M. V. A. de Moraes, and W. V. Matricardi are with the Department of Cartography, São Paulo State University, Presidente Prudente 19060-900, Brazil (e-mail: tomaseli@fct.unesp.br; galo@ fct.unesp.br; antunesdemoraes@gmail.com; matricardi.wander@gmail.com).

T. T. dos Reis is with SensorMap Company, Assis 19806-060, Brazil (e-mail: tiedtke@sensormap.com.br).

R. da S. Ruy is with Engemap Company, Assis 19806-060, Brazil (e-mail: roberto@engemap.com.br).

Color versions of one or more of the figures in this letter are available online at http://ieeexplore.ieee.org.

Digital Object Identifier 10.1109/LGRS.2017.2779559 ease of acquisition and documentation. Examples of data sets used in studies of computer vision and image processing can be found in [1] and [2].

In the field of remote sensing, the GRSS-IEEE provides access to several data sets that have been used in scientific contests [3]. For instance, the participants in the 2013 contest on image fusion used a data set that consists of a hyperspectral image and a LiDAR-derived digital surface model (DSM) that have the same spatial resolution $(2.5 \mathrm{~m})$ [4]. The 2015 contest used very high-resolution multisensor data derived from airborne RGB orthoimages with a 5-cm ground sampling distance (GSD), airborne laser scanning (ALS) data with an average density of 65 points $/ \mathrm{m}^{2}$, and a DSM with a point separation of $10 \mathrm{~cm} \mathrm{[5].} \mathrm{Other} \mathrm{data} \mathrm{sets} \mathrm{can} \mathrm{be} \mathrm{found} \mathrm{on} \mathrm{the}$ GRSS-IEEE website. Some of these data sets were released solely for researchers participating in specific contests. One such data set is composed of hyperspectral images with ground truth information [6].

The ISPRS also hosts several data sets, including those associated with technical benchmarks. Freely available data sets can be found in [7], including terrestrial-, aerial-, and satellite-related data. Scanned aerial images of the French region of Avenches were collected by ETH Zurich and are available in [8]. Another data set with aerial imagery is also available in [9]. Benchmark data sets are also distributed by EUROSDR for use in research on dense image matching [10].

The complexity associated with producing reference data for certain benchmarks was addressed by Wang et al. [11], who introduced a data set covering Toronto, Canada, using different sources of high-precision maps and images to produce ground truth data. To align these data, an automatic dataflow was developed.

Data sets can also be extracted from governmental spatial data infrastructures. The government of New Brunswick, for instance, offers full access to LiDAR data [12] through a web portal.

In addition, the Rhode Island Geographic Information System website provides access to geospatial data for Rhode Island, and it is maintained by the University of Rhode Island [13].

In Brazil, some state governments provide free access to geospatial data, including ALS point clouds. One example is Pernambuco, Brazil [14].

Even with these initiatives, there exists a lack of highresolution, multisensor, multiscale, and temporal geospatial databases for urban areas in tropical countries. Tropical areas 
display several differences when compared to existing data sets consisting of aerial imagery or of ALS of temperate regions, which are as follows.

1) Many more trees exist in scenes of tropical areas, in which canopies are dense and covered by leaves throughout the entire year, and there is a growing need for studies that examine urban trees [15].

2) The illumination by the sun provides more energy, and the sun elevation angle is sometimes at the nadir. This condition is impossible in temperate regions, from which most data sets originate.

3) The urban networks are heterogeneous, and the buildings are arranged in a much less structured way, leading to very complex environments.

4) The rate of change is quite high, and techniques that are suitable for performing automatic change detection are of great value.

Thus, data sets from tropical areas will be useful for evaluation of existing algorithms, which are usually tested with imagery from temperate regions. The data set presented in this letter contains high-resolution images collected at different flight heights and ALS data with different densities. A technical description of the equipment used in the data collection, the data to be distributed, and some assessment results are presented in the following sections.

\section{Equipment Used to Collect the Data Set}

The flight was performed for a periodic calibration of the data collection systems (both camera and ALS) and a geometric assessment of the aerial imaging platform used by the Engemap Company over a test area within Presidente Prudente, São Paulo, Brazil, that was prepared with its own resources, aircraft, and equipment. The ground control, data processing, and analysis of the results were performed by the SensorMap Company and the Unesp Photogrammetric Research Group.

A Piper Seneca III twin engine aircraft [Fig. 1(a)] was used. This aircraft includes a full set of equipment for the simultaneous acquisition of ALS and digital images with accurate trajectory estimation [Fig. 1(b)] acquisition. For this flight, some signalized targets [shown in Fig. 4(b)] were used as ground control points (GCPs).

\section{A. Main Features of the Sensors and Aircraft}

The ALS unit is an RIEGL LMS-Q680i. It uses multiple time around (MTA) processing and online digitizing of echo pulses and thereby enables postprocessing with full waveform analysis. Due to the MTA processing, even target echo signals that are outside of the unambiguity range between two sequential laser pulses can be processed and used [16]. This system uses the principles of LiDAR to measure distances from the unit to the ground with a precision of up to $2 \mathrm{~cm}$. It has a laser pulse repetition rate of up to $400 \mathrm{kHz}$ and an effective measurement rate of up to $266 \mathrm{kHz}$ at a $60^{\circ}$ scan angle. Accurate time synchronization is achieved through an interface with a Global Navigation Satellite System (GNSS) receiver, which provides Pulse-per-second signal (PPS) and National Marine Electronics Association (NMEA) streams. The coverage angle can be configured to view up to $60^{\circ}$. Mounting

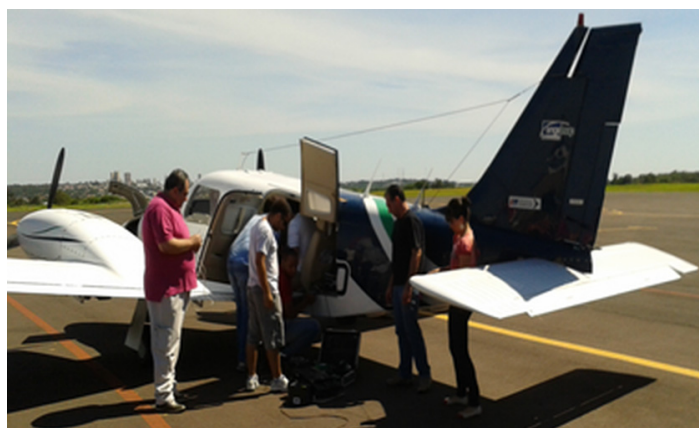

(a)

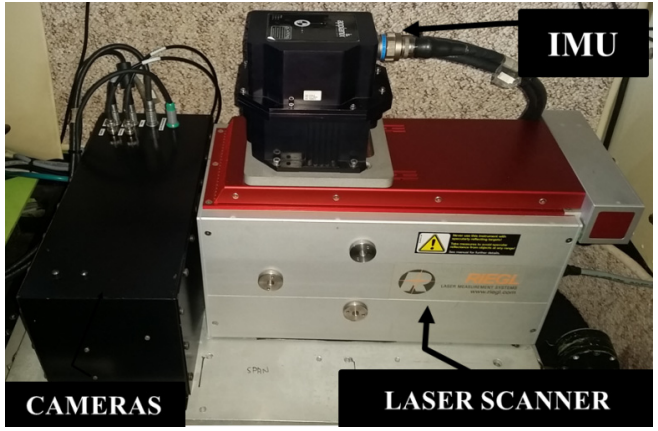

(b)

Fig. 1. (a) Piper Seneca III aircraft being prepared for flight. (b) View of the equipment installed inside the aircraft.

parameters and further calibration data are also required to provide accurate point cloud estimation. The precise trajectory was generated by postprocessing the data acquired by an integrated INS Applanix POS AV [17]. This system uses a dual frequency PGPS16 GNSS receiver integrated into an inertial measurement unit (IMU) model n. 31. The precision of the postprocessed positions ranges from 5 to $30 \mathrm{~cm}$, and the precision of the attitude angles ranges from $0.005^{\circ}$ for the roll and pitch and $0.008^{\circ}$ for the heading.

The digital images were acquired by a Phase One medium format camera (model iXA 180), which uses SchneiderKreuznach lenses with nominal focal lengths of $55 \mathrm{~mm}$. An image size of $10328 \times 7760$ pixels (80 megapixels) is acquired by a sensor with an effective size of $53.7 \mathrm{~mm} \times$ $40.4 \mathrm{~mm}$ with a coverage angle of $62^{\circ}$, resulting in a pixel size of $5.2 \mu \mathrm{m}$. A Bayer filter is used to produce three RGB bands. This camera has a time delay integration controller to minimize blurring, but its use was not necessary because the exposure time and lens aperture were adjusted to minimize motion blur, considering the illumination conditions on the day of the flight. The shutter for the lenses is made of a carbon fiber material capable of exposures as brief as $1 / 2500 \mathrm{~s}$, which enables low flight heights. Infrared images were also acquired by a 60-megapixel Hasselblad H4D camera with a nominal focal length of $50 \mathrm{~mm}$. These images will be made available in the future update of this data set. The RAW images were processed using Capture One software, and an automatic radiometric adjustment was performed without vignetting or distortion corrections. The converted RAW images were stored as 8-bit TIFF-LZW lossless compression files. 
TABLE I

Main Features of the Flight and Image Data

\begin{tabular}{|cccccc|}
\hline$\#$ & $\begin{array}{c}\text { Flight height } \\
\text { AGL }(\mathrm{m})\end{array}$ & Photo scale & $\begin{array}{c}\text { GSD } \\
(\mathrm{cm})\end{array}$ & $\begin{array}{c}\text { Dimensions } \\
(\mathrm{m} \mathrm{x} \mathrm{m})\end{array}$ \\
\hline 1 & 550 & $1: 10000$ & 5 & 537 & 404 \\
2 & 900 & $1: 16000$ & 8 & 859 & 646 \\
3 & 1100 & $1: 20000$ & 10 & 1074 & 807 \\
4 & 1300 & $1: 23636$ & 12 & 1269 & 954 \\
5 & 1900 & $1: 34545$ & 18 & 1855 & 1394 \\
\hline \hline \multicolumn{7}{c}{ Camera and Image Features } \\
\hline Nominal Camera & Terrain Mean & $\begin{array}{c}\text { Pixel } \\
\text { Size } \\
(\mathrm{mm})\end{array}$ & Number of & Columns \\
Focal Length (mm) & Elevation (m) of & Rows \\
\hline \multicolumn{7}{c}{55} & 420 & 0.0052 & 10328 & 7760 \\
\hline
\end{tabular}

\section{Data Set Description}

Due to the large size of the full set of data acquired, a reduced number of images was selected, and the point clouds were cropped to cover the area around the Unesp campus. The selected area, which has approximate dimensions of $900 \mathrm{~m} \times 1200 \mathrm{~m}$, covers the Unesp campus at Presidente Prudente and its surroundings, including a shopping mall, two football courts, houses, vegetated areas, and small water bodies. A variety of interesting features exist within this area, including buildings of different sizes, shapes, and heights, floor materials, and use types (e.g., commercial, residential, educational, industrial, and public facilities). The vegetation is composed of different species of trees with different heights, as well as grass and shrubs. The streets are mostly covered by black asphalt pavement, but they have different widths and are mostly covered by trees, thus creating a complex scenario for automatic algorithms. Additionally, the relief variation is approximately $50 \mathrm{~m}$, making this area suitable for testing different algorithms for feature extraction and classification, e.g., buildings, roads, trees, and water bodies, as well as for testing processes for data fusion, change detection, and reconstruction.

The flight was performed on December 16, 2014. Five different flight heights and cross strips along the backward and forward directions were used (Table I). With this setup, 436 images and 15 ALS point clouds were collected for different coverage areas and GSDs, depending upon the flight height.

Table I shows the flight heights above the mean ground level, the approximate photo scale, the GSD, and the dimensions covered by each image over the terrain. The aircraft trajectory with marked camera stations is shown in Fig. 2. The images were taken with an average shutter speed (exposure time) of $1 / 1000 \mathrm{~s}$ with ISO 50 and F5.6. The ALS was configured to use a coverage angle of $60^{\circ}$ and a laser pulse repetition rate of $400 \mathrm{kHz}$. From the complete set of 436 images, 14 were selected along two strips running in the $\mathrm{S}-\mathrm{N}$ and $\mathrm{N}-\mathrm{S}$ directions, which are depicted in the mosaics of Fig. 3. These images were acquired at a flight height of $900 \mathrm{~m}$ above the ground with a forward overlap of $80 \%$ and a GSD of $8 \mathrm{~cm}$.

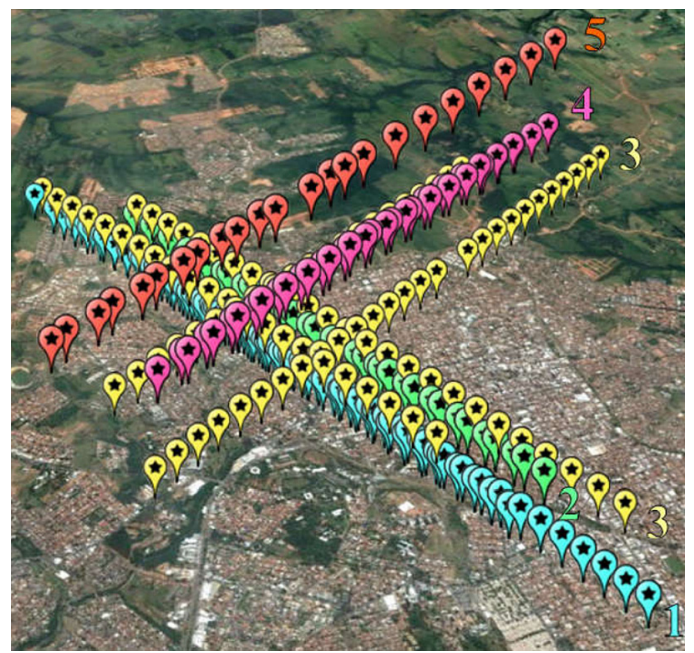

Fig. 2. Flight lines and camera stations at different flight heights. (Source: Google Earth).

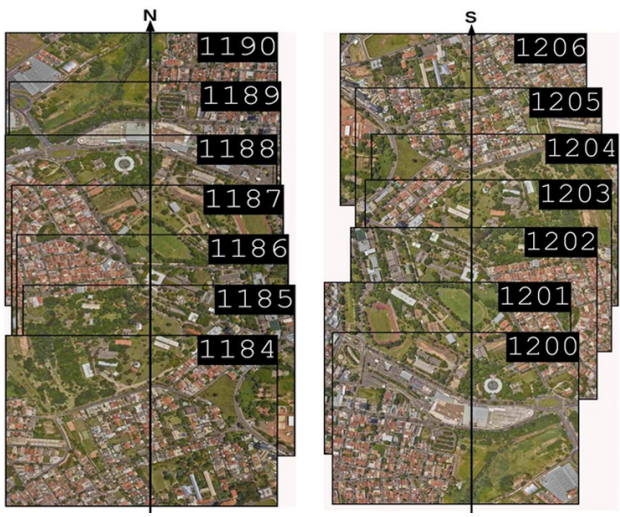

Fig. 3. Selected image strips in the north and south directions.

TABLE II

IOPs Estimated Using In Situ Calibration

\begin{tabular}{lcc}
\hline Parameter & Parameter value & Standard deviation \\
\hline Focal length $(\mathrm{mm})$ & 55.1281 & 0.0015 \\
$\mathrm{x}_{0}(\mathrm{~mm})$ & -0.1128 & 0.0014 \\
$\mathrm{y}_{0}(\mathrm{~mm})$ & 0.0612 & 0.0014 \\
$\mathrm{~K}_{1}\left(\mathrm{~mm}^{-2}\right)$ & $-2.43530 \mathrm{E}-05$ & $6.2 \mathrm{E}-08$ \\
$\mathrm{~K}_{2}\left(\mathrm{~mm}^{-4}\right)$ & $1.13684 \mathrm{E}-08$ & $1.3 \mathrm{E}-10$ \\
$\mathrm{~K}_{3}\left(\mathrm{~mm}^{-6}\right)$ & $-1.21236 \mathrm{E}-13$ & $8.6 \mathrm{E}-14$ \\
$\mathrm{P}_{1}\left(\mathrm{~mm}^{-1}\right)$ & $3.30591 \mathrm{E}-06$ & $1.7 \mathrm{E}-07$ \\
$\mathrm{P}_{2}\left(\mathrm{~mm}^{-1}\right)$ & $1.44741 \mathrm{E}-06$ & $1.7 \mathrm{E}-07$ \\
\hline
\end{tabular}

From the set of 15 point clouds, three were selected and cropped. Fig. 5 shows the three selected point clouds, and Fig. 6 shows the three cropped sections.

Owing to the use of direct georeferencing procedures, accurate position and attitude data for each camera station are available. Using these data and some GCPs, an in situ calibration was carried out with InBlock Inpho Software, version 5.4.1 [18], to produce accurate and reliable interior orientation parameters (IOPs). The values of these parameters are presented in Table II.

The data collected with the GNSS receiver and the IMU were postprocessed with POSPAC 6.0, a software package also 


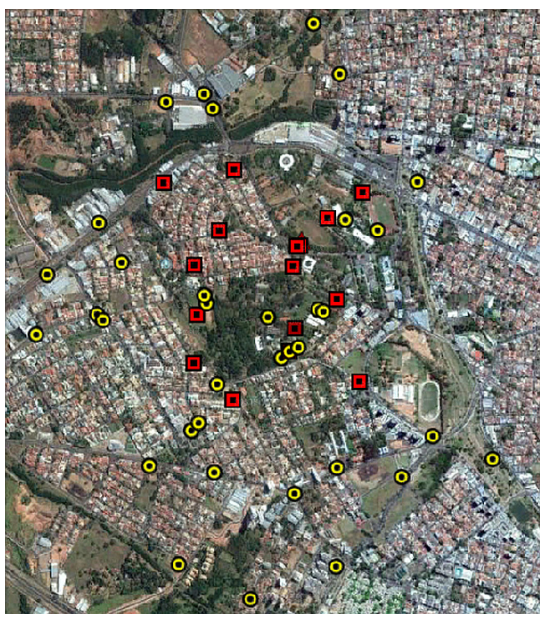

(a)

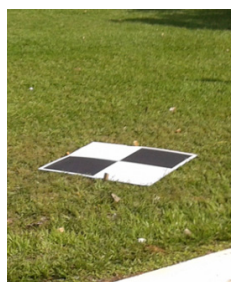

(b)

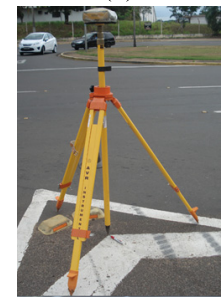

(c)
Fig. 4. (a) Distribution of GCPs over an area. Red points are signalized targets, whereas yellow points are natural. (b) One of the signalized targets and (c) natural point with a GNSS receiver.

provided by Applanix, using the PPTE reference base station of the Brazilian Continuous Monitoring Network (RBMC), which is located on the campus. The estimated standard deviation of the $\mathrm{E}$ and $\mathrm{N}$ coordinates of the camera station is approximately $1 \mathrm{~cm}$, while that of the $Z$ coordinate is approximately $2 \mathrm{~cm}$, and the standard deviations of the attitude angles are approximately $0.2^{\prime}$ for roll and pitch and $0.5^{\prime}$ for heading.

A set of 31 GCPs were surveyed with GNSS receivers via relative positioning and employing station PPTE of the GNSS RBMC as a reference base station [Fig. 4(a)]. For each point, a session of at least $20 \mathrm{~min}$ in length was performed. Within this control set, 17 GCPs had been previously signalized and surveyed in December 2014, as indicated by the targets shown in Fig. 4(b). Another set of GCPs surveyed in 2013 over existing features [Fig. 4(c)] was also considered for use in this project. All coordinates were determined within the SIRGAS2000 reference system, which is compatible with the WGS-84 reference system. The coordinates are available both in geodetic coordinates $(\varphi, \lambda, h)$ and in a UTM projection (zone 22 , central meridian: $\lambda_{\mathrm{CM}}=51 \mathrm{~W}$ ) with a standard deviation of approximately $2 \mathrm{~mm}$. Detailed descriptions of these points were also inserted into the data set. A KML file that displays the location and at least one image of each GCP is provided to the users.

The ALS data were processed using RIEGL software, and 15 point clouds were generated with different densities and five pulse returns. Boresight misalignment angles between the laser unit and the IMU were computed by the manufacturer using a calibration flight that had been carried out by Engemap in 2012. Fig. 5 presents three point clouds covering the entire flight area, and Fig. 6 shows the cropped areas that were selected for inclusion in this data set and are available for download. The colors were assigned as a function of the elevation and intensity of the returned pulse. Fig. 6(a) shows strip n. 121107, which was acquired from a flight height of $1300 \mathrm{~m}$ and presents an average point density of 2.9 points $/ \mathrm{m}^{2}$.

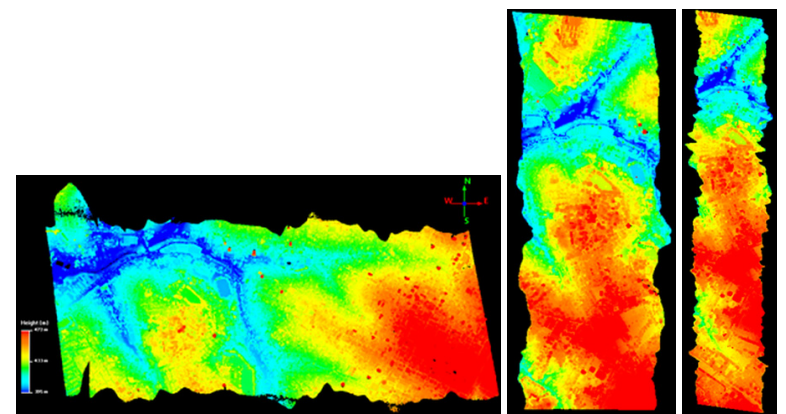

(a)

(b)

(c)

Fig. 5. Point clouds collected by ALS for three flight heights and three densities. (a) $1300 \mathrm{~m}$ with 2.9 points $/ \mathrm{m}^{2}$. (b) $900 \mathrm{~m}$ with 5.8 points $/ \mathrm{m}^{2}$. (c) $550 \mathrm{~m}$ with 12.5 points $/ \mathrm{m}^{2}$

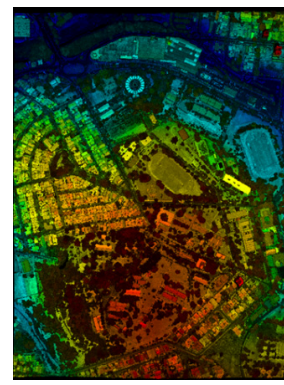

(a)

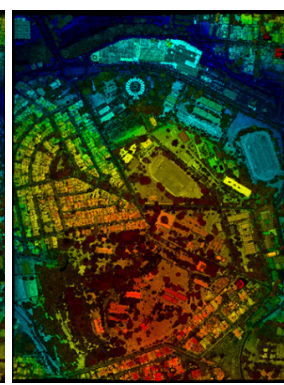

(b)

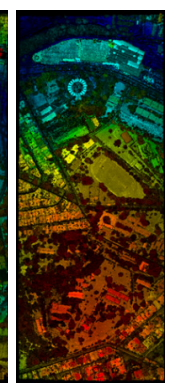

(c)
Fig. 6. Cropped point clouds for (a) strip n. 121107 with 2.9 points $/ \mathrm{m}^{2}$, (b) strip n. 120021 with 5.8 points $/ \mathrm{m}^{2}$, and (c) strip n. 132243 with 12.5 points $/ \mathrm{m}^{2}$.

Fig. 6(b) depicts strip n. 120021, which was acquired from a flight height of $900 \mathrm{~m}$ with an average point density of 5.8 points $/ \mathrm{m}^{2}$. Finally, Fig. 6(c) displays strip n. 132243, which was acquired from a flight height of $550 \mathrm{~m}$ and displays a density of 12.5 points $/ \mathrm{m}^{2}$.

\section{QuALity Metrics}

The selected set of images was used in a bundle adjustment (BA) in which the direct georeferencing parameters (camera positions and attitude angles) acquired by the GNSS and the IMU during the flight were used as initial values with constraints based on the estimated standard deviations. This BA was intended to check the consistency of the directly measured exterior orientation parameters (EOPs), the estimated IOPs, and the GCP coordinates.

The BA was performed in ERDAS Imagine-LPS using direct orientation data as constraints for the coordinates of the perspective centers (PCs) and as initial estimates of the attitude angles. The standard deviations of the PCs were set to $\sigma=5 \mathrm{~cm}$ for the three components, and the attitude angles were considered to be unknown. The standard deviations of the image coordinates were set to $\sigma=0.5$ pixel, and those of the ground coordinates were set to $\sigma=5 \mathrm{~cm}$. Tie points were automatically generated to be regularly distributed, with a default number of 25 points per model. The GCPs were measured manually in one image and transferred by least-squares matching to their homologous areas in other images. The a posteriori sigma (sigma naught) value was 0.4 for an a priori 
TABLE III

RMSES OF THE GCP AND CHECKPOINT COORDINATES. (A) COORDINATES ESTIMATED USING THE BA. (B) RMSEs OF THE COORDINATES CALCUlATED B F FORWARD INTERSECTION FOR THE CHECKPOINTS

\begin{tabular}{|c|c|c|c|c|c|}
\hline \multicolumn{3}{|c|}{$\begin{array}{c}\text { (A) RMSE of } 5 \text { GCPs estimated } \\
\text { through the BA }\end{array}$} & \multicolumn{3}{|c|}{$\begin{array}{l}\text { (B) RMSE of } 12 \\
\text { checkpoints }\end{array}$} \\
\hline$X(m)$ & $Y(\mathrm{~m})$ & $Z(m)$ & $X(m)$ & $Y(m)$ & $Z(\mathrm{~m})$ \\
\hline 0.03 & 0.03 & 0.05 & 0.04 & 0.04 & 0.07 \\
\hline
\end{tabular}

value of 1 , and the RMSEs of the residuals for the GCPs after the BA using five GCPs for both the $x$ and $y$ image coordinates were 0.26 pixel. Table III(a) presents the RMSEs in the ground coordinates resulting from the differences between the ground coordinates of the surveyed GCPs and those computed in the BA. The coordinates of 12 checkpoints were calculated by forward intersection, and the corresponding RMSEs are presented in Table III(b). In both cases, the RMSEs are smaller than the GSD.

The EOPs estimated using the BA were compared to the values measured directly using the GNSS and the IMU, and the RMSEs of the discrepancies in the camera station coordinates are 8,3 , and $3 \mathrm{~cm}$ in the $X-, Y$-, and $Z$-axes, respectively. A second test was performed using the BA without constraints in the PCs. The RMSEs associated with the GCPs and checkpoints were similar to those presented in Table III, and the RMSEs of the discrepancies in the coordinates of the PCs with respect to the values measured using GNSS were approximately 28,33 , and $6 \mathrm{~cm}$ in the $X$-, $Y$-, and $Z$-directions, respectively. The discrepancies were likely larger because the estimated EOPs absorbed the residual errors in the IOPs.

The cropped point cloud with a density of 5.8 points $/ \mathrm{m}^{2}$ was evaluated using the GCPs as a reference. For this analysis, only points on the ground were considered, and the elevation values corresponding to the planimetric $(\mathrm{E}, \mathrm{N})$ components were interpolated and compared with the elevations of the GCPs. The RMSE of the $Z$ component is $0.12 \mathrm{~m}$, which is compatible with the nominal vertical accuracy of the ALS.

\section{How to Use This Data Set}

This data set can be freely used for research with proper citation and credit to this letter and to Engemap Company, which provided the original data. It can be downloaded from the IEEE Dataport [19] or from the following URL:

http://www.fct.unesp.br/unespdataset

The e-mail address of the user will be used as the access key, and a password will be sent to this address to maintain a level of security. The user can then download the data. An e-mail address can be used to report problems and ask questions.

This data set is intended to be dynamically expanded. Existing multitemporal data (both ALS and digital images, including scanned historical images) will be incorporated in the future, including flights with similar characteristics. Generating reliable ground truth data is more challenging, but the data set is expected to include vector layers, in addition to geometric control information. Suggestions and contributions from users in this matter will be greatly appreciated. Very high-resolution hyperspectral and RGB images acquired by sensors onboard remotely piloted aerial systems will also be included.

\section{CONCLUSION}

Current data acquisition capacities are massive. Paradoxically, however, there is a lack of well-tested, freely distributed multisensor, multitemporal, and multiresolution data sets that are tailored for scientific studies that primarily focus on complex urban environments, such as those in tropical areas. This data set is primarily intended to be a scalable resource that will grow to contain data originating from different sources and epochs from a tropical area containing several different types of features. It is expected that researchers will use these data to test their algorithms, produce novel approaches, and provide feedback to improve future versions of the data set described in this letter.

\section{ACKNOWLEDGMENT}

The authors would like to thank R. Garcia for his kind support in preparing the webpage for this data set.

\section{REFERENCES}

[1] G. Mazars. (2017). CV Datasets on the Web. [Online]. Available: http:// www.cvpapers.com/index.html

[2] A. Sarafraz. (2017). Computer Vision On Line-Data Sets. [Online]. Available: http://www.computervisiononline.com/datasets

[3] GRSS Society. (2017). Image Analysis and Data Fusion. [Online]. Available: https://www.grss-ieee.org/community

[4] C. Debes et al., "Hyperspectral and LiDAR data fusion: Outcome of the 2013 GRSS data fusion contest," IEEE J. Sel. Topics Appl. Earth Observ. Remote Sens., vol. 7, no. 6, pp. 2405-2418, Jun. 2014.

[5] M. Campos-Taberner et al., "Processing of extremely high-resolution LiDAR and RGB Data: Outcome of the 2015 IEEE GRSS data fusion contest-Part A: 2-D contest," IEEE J. Sel. Topics Appl. Earth Observ. Remote Sens., vol. 9, no. 12, pp. 5547-5559, Dec. 2016.

[6] N. Acito, S. Matteoli, A. Rossi, M. Diani, and G. Corsini, "Hyperspectral airborne 'viareggio 2013 trial' data collection for detection algorithm assessment," IEEE J. Sel. Topics Appl. Earth Observ. Remote Sens., vol. 9, no. 6, pp. 2365-2376, Jun. 2016.

[7] ISPRS. (2017). ISPRS Dataset. [Online]. Available: http://www.isprs. org/data/default.aspx

[8] Institute of Geodesy and Photogrammetry, ETH Zurich. (2017) Avenches (France) Data Set. [Online]. Available: http://www.isprs.org/ data/avenches/default.aspx

[9] Institute of Geodesy and Photogrammetry, ETH Zurich. (2017). Zurich Hoengg (Switzerland) Data Set. [Online]. Available: http://www. isprs.org/data/hoengg

[10] N. Haala et al. (2016). Benchmark on High Density Aerial Image Matching. [Online]. Available: http://www.ifp.uni-stuttgart.de/ISPRSEuroSDR/ImageMatching/index.en.html

[11] S. Wang et al. (2016). "TorontoCity: Seeing the world with a million eyes." [Online]. Available: https://arxiv.org/abs/1612.00423

[12] Government of New Brunswick. (2017). Lidar Inventory. [Online]. Available: http://geonb.snb.ca/li/index.html

[13] University of Rhode Island. (2017). Rhode Island Geographic Information System (RIGIS). [Online]. Available: http://www.rigis.org/

[14] Governo do Estado de Pernambuco. (2017). Pernambuco 3D. [Online]. Available: http://www.pe3d.pe.gov.br/index.php

[15] J.-P. Rossi, V. Imbault, T. Lamant, and J. Rousselet, "A dataset on the inventory of coniferous urban trees in the city of Orléans (France)," Data Brief, vol. 9, pp. 803-806, Dec. 2016.

[16] Riegl. (2017). Airborne Laser Scanner. [Online]. Available: http://www. riegl.com/products/airborne-scanning

[17] Applanix. (2017). Applanix POS AV 510. [Online]. Available: https:// www.applanix.com/pdf/posav_specs_1212.pdf

[18] Inpho Trimble. (2017). GeoReferencing Inpho Software. [Online]. Available: http://www.trimble.com/Imaging

[19] A. Tommaselli et al. (2017). A Dataset Containing Frame Images and Dense Airborne Laser Scanning Point Clouds. [Online]. Available: http://dx.doi.org/10.21227/H2SK8C 